\title{
The Journal and Guidelines
}

\author{
Eelco F. M. Wijdicks ${ }^{1}$
}

Published online: 21 December 2015

(c) Springer Science+Business Media New York 2015

How often do you use a guideline when deciding on the best practice in your patient? I would certainly hope you do frequently. There are those who argue there is limited success of clinical practice guidelines in changing decision making - or even changing an attitude. In the extreme position, guidelines are considered to create uncertainty where certainty supposedly existed before and it may determine policy. And then of course the question begs who is the expert panel and on the bases of what qualifications? Interpretation of a guideline as an undisputed directive or even standard of care is part of the problem. Guidelines are provided as an educational service to practicing neurointensivists and adherence to guidelines remains voluntary.

I have been closely involved in over half dozen guidelines and scientific statements and know criticism first hand. And yet, I also know it is very, very hard work requiring a laser focus of the authors to get it right. In our field, we need more scientific statements and consensus reports even if this is what we need to do until we have better data. Guidelines it must provide fair and reliable recommendations and eventually allows evidence-based decision making during patient care.

The journal is the perfect vehicle for publication of such guidelines and scientific statements, and we have published several in the past, and made it easy accessible for subscribers.

Eelco F. M. Wijdicks

wijde@mayo.edu

1 Division of Critical Care Neurology, Mayo Clinic, Rochester, USA
In this first issue of 2016, we open with three new guidelines endorsed by the Neurocritical Care Society. These new guidelines demand attention because the topics are on common complex decisions and a comprehensive critical review perusing all relevant papers had not been addressed before. These Neurocritical Care Society guidelines also demonstrate a continuous advancement in vetting of the literature, consensus building, and writing by the committee.

In each of these guidelines, a committee of experts was recruited from within the Neurocritical Care Society. A method of generating clinical questions was used and divided into four components, the so-called Oxford PICO questions $(P$ Population/patient, $I$ Intervention/indicator, $C$ Comparator/control, $O$ Outcome). The committees utilized GRADE methodology to adjudicate the quality of evidence as high, moderate, low, or very low based on their confidence that the estimate of effect was close to the true effect. They generated recommendations only after considering quality of evidence, relative risks and benefits, patient values and preferences, and resource allocation.

The committee faced a common concern: the paucity of evidence. Nonetheless, recommendations could be distilled from a large body of literature sometimes with personal opinion seeping through in conclusions labeled as "strong recommendation, low quality evidence."

One guideline involves the insertion and management of ventriculostomies. There are considerable differences in management and concerns over the rate of complications such as infection, malposition, and hemorrhage. The paper provides statements about thromboembolism prophylaxis, antimicrobial impregnated catheters, and how to best adhere to a bundle of care. 
The second guideline involves reversal of anticoagulation in cerebral hemorrhage. In conjunction with the Society of Critical Care Medicine, an international committee with a diverse group of experts provides recommendations for the reversal of warfarin, direct factor $\mathrm{Xa}$ antagonists, direct thrombin inhibitors, unfractionated heparin, low molecular weight heparin, thrombolytics, and also antiplatelet agents. Resumption of anticoagulation-a much more nebulous area-will be addressed in a future guideline.

The third guideline is on prophylaxis of venous thrombosis. The goal of this guideline is to provide an evidencebased manuscript to address the appropriate administration of thromboprophylaxis (pharmacological and mechanical prophylaxis) in patients with neurologic illness and thus the paper specifically addresses decisions in patient with ischemic stroke, intracranial and intraventricular hemorrhage, aneurysmal subarachnoid hemorrhage, traumatic brain injury, spinal cord injury, brain tumors, acute neuromuscular disorders, and patients undergoing neurosurgical and neurovascular interventions.

The Institute of Medicine has set standards for trustworthy guidelines. These standards demand the most up to date literature, expert consensus, and public input. The standards include establishing transparency, management of conflict of interest, guideline development group composition, clinical practice guideline-systematic review intersection, establishing evidence foundations for rating strength of recommendations, articulation of recommendations, external review, and updating (iom. nationalacademies.org). The membership should be proud of the guideline committee to meet these standards and to keep us informed. Changing practice based on good evidence is good practice.

This year will mark another year with new innovative features. I am pleased to have Stephan Mayer back on board. He has been a major force from day one and will assume the role of associate editor of current concept papers. In addition, this year we will be starting a neurocritical care fellow section where fellows and residents becoming fellows can submit their papers. This section will focus on particular difficult decisions faced by fellows often in the dead of night.

The journal progressed as planned. All the more so, we will publish more manuscripts and continue to expand the number of supplements. The journal welcomes prospective clinical trials and high quality topic reviews and we move it forward quickly. Our time from submission to decision is very short-measured in days - and our review timemeasured in a few weeks-is among the best of any published journal. 